\title{
Spécialistes en pharmacologie et toxicologie cliniques en Suisse
}

Mitsuko Kondo-Oestreicher

Correspondance:

Dr Mitsuko Kondo-Oestreicher HUG

Pharmacologie et

toxicologie cliniques

Rue Gabrielle-Perret-Gentil 4

1211 Genève 14

Tél. 0223729004

mitsuko.kondo-oestreicher@

hcuge.ch

www.clinpharm.ch

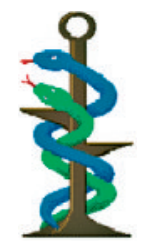

Les médecins, pharmaciens et autres titulaires d'un doctorat en sciences ayant suivi une formation de spécialistes en pharmacologie et toxicologie cliniques exercent en milieu hospitalier, dans des institutions publiques, auprès des autorités sanitaires, dans l'industrie ou encore, en pratique privée. Les médecins spécialisés en pharmacologie clinique ont suivi une formation d'au moins 3 ans en médecine interne, pédiatrie ou anesthésiologie, puis une formation spécialisée de 3 années supplémentaires [1]. Les pharmaciens et les titulaires d'un doctorat en sciences doivent pour leur part suivre une formation en pharmacologie clinique d'au moins 4 ans après l'obtention de leur doctorat.

\section{Pharmacologie clinique: \\ améliorer la qualité des soins}

Comme ailleurs dans le monde, la mission de la pharmacologie clinique suisse est d'améliorer les soins prodigués aux patients en favorisant l'utilisation efficace, rationnelle, adaptée, sûre et maîtrisée (ERASM) des médicaments. Plusieurs activités sont déployées à cette fin, comme l'évaluation des nouveaux traitements et leur introduction auprès des patients. Pour se faire, les spécialistes de la pharmacologie clinique s'appuient sur divers outils conceptuels comme la pharmacocinétique, la pharmacodynamique, la pharmacogénétique, la pharmacoépidémiologie, la pharmacovigilance, la pharmacométrie, la pharmaco-économie et la pharmacologie sociale. Les pharmacologues cliniciens interviennent aussi auprès de différentes instances cliniques nationales. A l'échelle locale, ils peuvent diriger les travaux des commissions chargées de l'évaluation des médicaments et produits thérapeutiques dans les établissements hospitaliers, établir et tenir à jour les listes de médicaments, évaluer les nouveaux produits, formuler des recommandations en matière de prescription et encadrer leur application, recenser les effets indésirables des médicaments et promouvoir la thérapeutique factuelle [2].

\section{Toxicologie clinique:}

\section{conseil et traitement des intoxications}

La toxicologie clinique est principalement investie d'une mission de conseil (auprès du Centre suisse d'information toxicologique et des centres universitaires régionaux de pharmacologie clinique), de traite- ment des intoxications et surdosages et d'évaluation des données relatives aux expositions accidentelles et délibérées aux médicaments, produits chimiques et plantes (toxicovigilance). A tous les niveaux, les pharmaco-toxicologues travaillent en étroite collaboration avec les autres professionnels de santé. Ainsi, ils sont fréquemment appelés à collaborer avec les prescripteurs ou médecins traitants et les patients pour le diagnostic et le traitement; ils participent à la dispensation des médicaments avec les pharmaciens d'officine ou ceux des hôpitaux/cliniques et les infirmières; ils surveillent l'action des médicaments avec les chimistes, biochimistes et biologistes; prennent part à la surveillance des effets indésirables des médicaments (pharmacovigilance et toxicovigilance) auprès des instances régionales, nationales et internationales et participent à des recherches cliniques aux côtés des investigateurs, moniteurs d'essais cliniques, membres des comités d'éthique et autorités sanitaires locales. Il leur appartient également d'exercer une mission de service public en diffusant des informations scientifiques indépendantes et utiles sur les médicaments et les produits chimiques.

\section{La Société suisse de pharmacologie et de toxicologie cliniques}

Les spécialistes en pharmacologie et toxicologie cliniques participent de très près aux programmes de formation de premier, deuxième et troisième cycles, et élaborent des outils et ressources d'enseignement pour optimiser la prescription des médicaments.

La formation est un domaine d'intervention essentiel de la SSPTC. La Société participe également à des recherches cliniques, à l'enseignement des bonnes pratiques cliniques, à des recherches translationnelles et à la diffusion de recommandations thérapeutiques fondées sur la médecine factuelle. Elle met aussi ses experts à la disposition du public et de différentes instances pour toutes questions en rapport avec les médicaments.

\section{Références}

1 www.fmh.ch/de/data/pdf/klinische_pharmakologie_ version_internet_f.pdf.

2 Consultant physicians working with patients. $4^{\text {th }}$ edition. London: Royal College of Physicians; 2008. 